\title{
Predictors of mortality in rheumatoid arthritis-associated interstitial lung disease
}

Joshua J. Solomon ${ }^{1}$, Jonathan H. Chung ${ }^{2}$, Gregory P. Cosgrove ${ }^{1}$, M. Kristen Demoruelle ${ }^{3}$, Evans R. Fernandez-Perez ${ }^{1}$, Aryeh Fischer ${ }^{3}$, Stephen K. Frankel ${ }^{1}$, Stephen B. Hobbs ${ }^{4}$, Tristan J. Huie ${ }^{1}$, Jill Ketzer ${ }^{1}$, Amar Mannina ${ }^{5}$, Amy L. Olson ${ }^{1}$, Gloria Russell ${ }^{6}$, Yutaka Tsuchiya $^{7}$, Zulma X. Yunt ${ }^{1}$, Pearlanne T. Zelarney ${ }^{8}$, Kevin K. Brown ${ }^{1}$ and Jeffrey J. Swigris ${ }^{1}$

Affiliations: ${ }^{1}$ Autoimmune Lung Center and Interstitial Lung Disease Program, National Jewish Health, Denver, CO, USA. ${ }^{2}$ Division of Radiology, National Jewish Health, Denver, CO, USA. ${ }^{3}$ Division of Rheumatology, University of Colorado School of Medicine, Aurora, CO, USA. ${ }^{4}$ Department of Radiology, University of Kentucky, Lexington, KY, USA. ${ }^{5}$ Creighton University School of Medicine, Omaha, NE, USA. ${ }^{6}$ Pontificia Universidad Católica Madre y Maestra, Santiago, Dominican Republic. ${ }^{7}$ Department of Respiratory Medicine, Saitama Cardiovascular and Respiratory Center, Kumagaya, Japan. ${ }^{8}$ Division of Biostatistics and Bioinformatics, National Jewish Health, Denver, CO, USA.

Correspondence: Joshua Solomon, Autoimmune Lung Center and Interstitial Lung Disease Program, National Jewish Health, Southside Building, Office \#G08c, 1400 Jackson Street, Denver, Colorado 80206, USA.

E-mail: SolomonJaNJHealth.org

ABSTRACT Interstitial lung disease (ILD) is a common pulmonary manifestation of rheumatoid arthritis. There is lack of clarity around predictors of mortality and disease behaviour over time in these patients.

We identified rheumatoid arthritis-related interstitial lung disease (RA-ILD) patients evaluated at National Jewish Health (Denver, CO, USA) from 1995 to 2013 whose baseline high-resolution computed tomography (HRCT) scans showed either a nonspecific interstitial pneumonia (NSIP) or a "definite" or "possible" usual interstitial pneumonia (UIP) pattern. We used univariate, multivariate and longitudinal analytical methods to identify clinical predictors of mortality and to model disease behaviour over time.

The cohort included 137 subjects; 108 had UIP on HRCT (RA-UIP) and 29 had NSIP on HRCT (RA-NSIP). Those with RA-UIP had a shorter survival time than those with RA-NSIP (log rank $\mathrm{p}=0.02)$. In a model controlling for age, sex, smoking and HRCT pattern, a lower baseline \% predicted forced vital capacity (FVC \% pred) (HR 1.46; p $<0.0001)$ and a $10 \%$ decline in FVC \% pred from baseline to any time during follow up (HR 2.57; $\mathrm{p}<0.0001)$ were independently associated with an increased risk of death.

Data from this study suggest that in RA-ILD, disease progression and survival differ between subgroups defined by HRCT pattern; however, when controlling for potentially influential variables, pulmonary physiology, but not HRCT pattern, independently predicts mortality.

@ERSpublications

In rheumatoid-arthritis associated interstitial lung disease, physiology, and not HRCT pattern, predicts mortality http://ow.ly/Uf1IF

This article has supplementary material available from erj.ersjournals.com

Received: March 042015 | Accepted after revision: Sept 202015 | First published online: Nov 192015

Support statement: JJ. Swigris is supported in part by a Career Development Award from the National Institutes of Health (K23 HL092227).

Conflict of interest: Disclosures can be found alongside the online version of this article at erj.ersjournals.com

Copyright OERS 2016 


\section{Introduction}

Rheumatoid arthritis is an autoimmune, inflammatory arthritis affecting $1 \%$ of the US population [1]. The lungs are the most common site of extra-articular involvement. Though rheumatoid arthritis can affect all compartments of the respiratory system (e.g. airways, pleura), its effects on the parenchyma result in the greatest morbidity [2]. Rheumatoid arthritis-related interstitial lung disease (RA-ILD) is identified in up to $60 \%$ of patients with rheumatoid arthritis, with clinically significant disease occurring in $10 \%[3,4]$.

The most common patterns of RA-ILD are usual interstitial pneumonia (UIP) and nonspecific pneumonia (NSIP) [5]. Patients with clinically significant RA-ILD and a UIP pattern on either high-resolution computed tomography (HRCT) or surgical pathology have a poor outcome, with survival estimates similar to patients with idiopathic pulmonary fibrosis (IPF) [6-8]. Available data suggest that patients with RA-NSIP have a more favourable prognosis than patients with RA-UIP [7].

A recently published expert review highlighted the paucity of quality data addressing predictors of outcome or describing longitudinal disease behaviour in patients with RA-ILD [9]. We sought to examine a group of patients with well-characterised RA-ILD to determine clinical predictors of mortality and to assess whether disease progression differs for those with UIP versus those with NSIP on HRCT.

\section{Methods}

Sample

This study was conducted at National Jewish Health (NJH) (Denver, CO, USA), a multi-specialty tertiary referral centre, and was approved by the NJH institutional review board (HS-2584). The NJH Research Database was queried, and all patients evaluated between 1995 and 2013 who had a rheumatologist-confirmed diagnosis of rheumatoid arthritis and HRCT evidence of ILD were considered for inclusion $(n=300)$. Patients with HRCT patterns other than UIP or NSIP, patients infected with atypical mycobacteria or those without HRCT scans available for physical review were excluded (see Figure S1 in the supplementary material for details). The first visit at NJH was used as their date of diagnosis. The final cohort included 137 subjects.

\section{Radiology}

Each subject's HRCT scan was independently reviewed by two expert thoracic radiologists (J.H. Chung and S.B. Hobbs) who were blinded to the subjects' clinical status and demographics. All disagreements were resolved through consensus. Scans were classified as showing a UIP (RA-UIP) or NSIP (RA-NSIP) pattern (see supplementary material for details) and UIP was further classified by degree of confidence ("UIP" or "possible UIP"). For the remainder of the manuscript, unless otherwise stated, RA-UIP refers to the subgroup with either UIP or possible UIP on HRCT; RA-NSIP refers to the subgroup with NSIP on HRCT.

\section{Data analysis}

We generated summary statistics for baseline characteristics. $\mathrm{p}<0.05$ was considered to represent statistical significance for all analyses. SAS Version 9.3 (SAS Inc., Cary, NC) was used to perform all analyses.

Survival analyses

We used the Kaplan-Meier method to display and the log-rank test to compare survival curves for the cohort stratified on HRCT pattern. We used Cox proportional hazards to examine HRCT pattern as a predictor of time-to-death while controlling for potentially influential variables. In certain analyses, we included a pulmonary physiology variable (e.g., forced vital capacity (FVC) \% predicted or diffusing capacity of the lung for carbon monoxide (DLCO) \% predicted) as a time-varying covariate; this allowed us to examine the effect of a 10-point drop in FVC \% pred or DLCO \% pred from baseline at any time during follow-up (e.g. a fall in FVC \% pred from $60 \%$ to 50\%). For retrospective studies, in which pulmonary physiology variables were obtained at the discretion of the treating physician and not at consistent time intervals (e.g. 3 or 6 months), the least bias may be imposed, and case-wise deletion can be avoided, by analysing variables as time-varying covariates. FVC \% pred and DLCO \% pred were strongly correlated $(\mathrm{r}=0.7)$; so, to avoid issues with multi-collinearity, we did not include both in the same model. Instead, we built multiple models that included one or the other and, because it has become the most widely used physiologic outcome variable in other forms of pulmonary fibrosis, we chose to focus on FVC \% pred. The Akaike Information Criterion was used to compare fit between models with lower values connoting better fit. Since including variables with substantial missing data can introduce significant bias, rheumatoid factor and anti-cyclic citrullinated protein (anti-CCP) antibodies, which both had a substantial number of missing values, were not included in multivariable models. Vital status was ascertained on July 31, 2015. No subjects were transplanted, so subjects were censored on July 31, 2015 if their death could not be confirmed in the Social Security Death Index. Three subjects without Social Security numbers were censored at the time of their last recorded clinic visits. 
Disease behaviour over time

To analyse disease behaviour (i.e. FVC \% pred or DLCO \% pred) over time, we used linear mixed-effects models, in which we allowed separate fits for subjects with UIP or NSIP. Each model included random terms for intercept and slope (for time-from-diagnosis) to account for the data structure (repeated measures over time within subject). In each model, the covariance for the repeated measures was left unstructured, as this yielded the best fits.

\section{Results}

Baseline characteristics for the cohort are presented in table 1. The median time from HRCT to baseline FVC \% pred was 0.0 days (interquartile range -1-151) for subjects with RA-UIP and 0.0 days (interquartile range -1-498) for subjects with RA-NSIP.

\section{Survival}

Over the follow-up period, 54 subjects died (47 (44\%) RA-UIP versus seven (24\%) RA-NSIP; Chi-squared 3.59, $\mathrm{p}=0.05$ ), and medial survival for the cohort as a whole was 10.35 (95\% CI 8.24-12.85) years. Subjects with RA-UIP had worse survival than those with RA-NSIP (median survival 10.18 years versus 13.62 years; $\log$ rank $\mathrm{p}=0.02$ ) (figure 1).

\begin{tabular}{|c|c|c|c|}
\hline Variable & All subjects & RA-UIP & RA-NSIP \\
\hline Subjects $n$ & 137 & 108 & 29 \\
\hline Age years & $64.7 \pm 10.6$ & $66.0 \pm 9.1$ & $60.1 \pm 14.3$ \\
\hline Female n (\%) & $68(50)$ & $52(48)$ & $16(55)$ \\
\hline \multicolumn{4}{|l|}{ Smoking history } \\
\hline Available $n$ & 135 & 106 & 29 \\
\hline Ever smokers n (\%) & $87(64)$ & 73 (69) & $14(48)$ \\
\hline Pack-years & $31.8 \pm 23.1$ & $33.6 \pm 24.1(n=67)$ & $20.0 \pm 11.5(n=12)$ \\
\hline Never smokers n (\%) & $48(36)$ & $33(31)$ & $15(52)$ \\
\hline \multicolumn{4}{|l|}{ Family history of RA } \\
\hline Available $\mathrm{n}$ & 123 & 94 & 29 \\
\hline Family history of RA n (\%) & $29(24)$ & $23(24)$ & $6(21)$ \\
\hline $\begin{array}{l}\text { Duration of RA preceding ILD years } \\
\text { RA medications }\end{array}$ & & $16.6 \pm 14.4$ \\
\hline Methotrexate ever n (\%) & $89(65)$ & $67(63)$ & $22(71)$ \\
\hline Biologic agent ever $\mathrm{n}(\%)$ & $87(64)$ & $64(60)$ & $23(74)$ \\
\hline \multicolumn{4}{|l|}{ FVC } \\
\hline Available $\mathrm{n}$ & 135 & 107 & 28 \\
\hline$\%$ pred & $69.3 \pm 19.2$ & $69.7 \pm 18.5$ & $67.8 \pm 21.9$ \\
\hline \multicolumn{4}{|l|}{ FEV 1} \\
\hline Available $\mathrm{n}$ & 134 & 106 & 28 \\
\hline$\%$ pred & $72.7 \pm 20.1$ & $73.5 \pm 19.5$ & $69.4 \pm 22.1$ \\
\hline \multicolumn{4}{|l|}{ D.co } \\
\hline Available $\mathrm{n}$ & 108 & 84 & 24 \\
\hline$\%$ pred & $48.9 \pm 17.8$ & $49.1 \pm 16.9$ & $48.3 \pm 20.7$ \\
\hline \multicolumn{4}{|l|}{ CPI } \\
\hline Available $\mathrm{n}$ & 106 & 83 & 23 \\
\hline$\%$ pred & $47.2 \pm 14.7$ & $47.0 \pm 13.9$ & $47.6 \pm 17.6$ \\
\hline \multicolumn{4}{|l|}{ Anti-CCP antibodies } \\
\hline Available $\mathrm{n}$ & 80 & 61 & 19 \\
\hline $\mathrm{CCP}>20 \mathrm{n}(\%)$ & $66(83)$ & $50(82)$ & $16(84)$ \\
\hline CCP titre for CCP positive & $140.5 \pm 87.1$ & $143.6 \pm 85.1$ & $130.5 \pm 95.0$ \\
\hline \multicolumn{4}{|l|}{ RF } \\
\hline Available $\mathrm{n}$ & 93 & 74 & 19 \\
\hline $\mathrm{RF}>20 \mathrm{n}(\%)$ & $78(84)$ & $64(86)$ & $14(74)$ \\
\hline RF titre for RF positive & $907.1 \pm 1754.7$ & $1054.0 \pm 1914.0$ & $336.8 \pm 462.3$ \\
\hline Died n (\%) & $54(39)$ & $47(43.5)$ & $7(24)$ \\
\hline Median (IQR) follow-up years & $4.8(2.5-7.8)$ & $4.6(2.4-7.7)$ & $6.9(4.1-8.1)$ \\
\hline
\end{tabular}

Data are presented as mean \pm SD, unless otherwise stated. RA: rheumatoid arthritis; UIP: usual interstitial pneumonia; NSIP: nonspecific interstitial pneumonia; ILD: interstitial lung disease; FVC: forced vital capacity; FEV1: forced expiratory volume in $1 \mathrm{~s} ; \mathrm{LLCO}$ : diffusing capacity of the lung for carbon monoxide; $\mathrm{CPI}$ : composite physiologic index; CCP: cyclic citrullinated peptide; RF: rheumatoid factor; IQR: interquartile range. 
FIGURE 1 Kaplan-Meier survival curve for patients with a high resolution computed tomography pattern of usual interstitial pneumonia (UIP) lany level of confidence) and nonspecific interstitial pneumonia (NSIP).

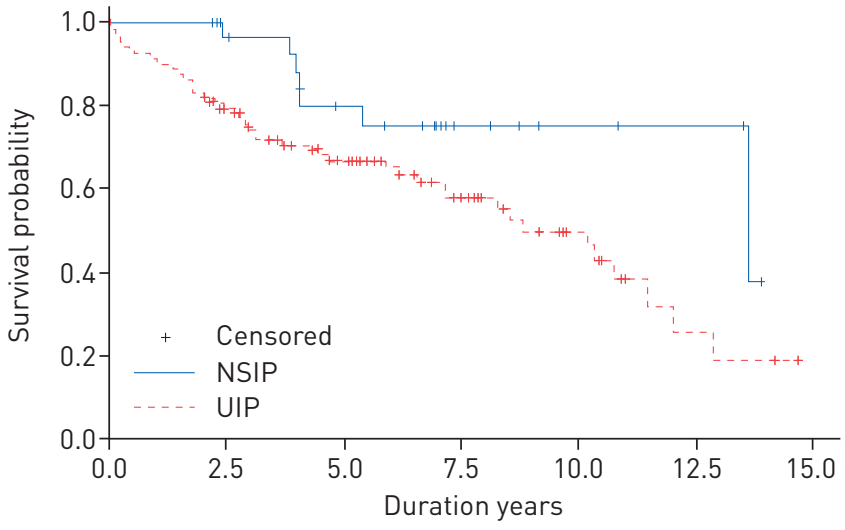

NSIP $29 \begin{array}{lllllllllllllll}29 & 29 & 24 & 23 & 18 & 16 & 13 & 8 & 6 & 5 & 4 & 4 & 4 & 0\end{array}$ UIP $108 \begin{array}{llllllllllllllll}108 & 89 & 69 & 59 & 48 & 39 & 32 & 23 & 19 & 14 & 7 & 5 & 3 & 3 & 0\end{array}$

Age, smoking history, baseline HRCT pattern, FVC \% pred, DLCO \% pred, a composite physiological index (CPI) and rheumatoid factor positivity were significant predictors of mortality in univariate time-to-death analyses (table 2). Results for multivariate survival analyses are presented in table 3 . While adjusting for age, sex and smoking, HRCT pattern was not significantly associated with survival (Model 2). While controlling for baseline and change over time in FVC \% pred, HRCT pattern was not a significant predictor of mortality (Model 4). In a model controlling for age, sex, smoking and both baseline FVC \% pred and change in FVC \% pred over time, HRCT pattern was not a significant predictor of mortality (Model 5). In that model, subjects with a $10 \%$ drop in FVC \% pred at any point during follow up died at a rate 2.5 -fold greater than for subjects whose FVC \% pred did not drop by $10 \%$ (HR 2.57; p $<0.0001$ ). A similar pattern was seen when DLCO \% pred replaced FVC \% pred in the models; for example, in a model that included HRCT pattern and both baseline and longitudinal change in DLCO $\%$ pred, subjects with a $10 \%$ drop in DLCO \% pred at any point during follow up died at a rate that was 1.3 -fold greater than for subjects whose DLCO \% pred did not drop by $10 \%$ (HR 1.34; $\mathrm{p}=0.02$ ). The interaction term (HRCT pattern $\times$ FVC $\%$ pred) was not significant (HR 1.03; $\mathrm{p}=0.3$ ) in a univariate analysis, so it was not included in multivariate models.

\section{Disease behaviour over time}

The slope of FVC \% pred was different between RA-UIP and RA-NSIP subgroups (difference in slopes UIP-NSIP $-2.11 \%$ per year, $95 \% \mathrm{CI}-4.16--0.07 ; \mathrm{p}=0.04$ ) (figure $2 \mathrm{a}-\mathrm{c}$ ). The slope of FVC $\%$ pred for the RA-UIP subgroup was $-0.83 \%$ per year, $95 \%$ CI $-1.79-0.13$; $\mathrm{p}=0.09$. The slope of DLCO $\%$ pred also differed between subgroups (difference in slopes UIP-NSIP $-3.10 \%$ per year, 95\% CI $-5.44--0.75 ; \mathrm{p}=0.01$ ) (figure $2 \mathrm{~d}-\mathrm{f}$ ). The slope of DLCO $\%$ for the RA-UIP subgroup was $-0.53 \%$ per year, $95 \%$ CI $-1.65-0.59 ; \mathrm{p}=0.60$.

TABLE 2 Univariate analyses for predictors of mortality

\begin{tabular}{lcc} 
Variable & HR (95\% CI) & p-value \\
\hline Age, year increase over mean 64.7 & $1.05(1.02-1.08)$ & 0.001 \\
Female sex & $0.58(0.34-1.02)$ & 0.06 \\
Ever-smoker & $2.58(1.36-4.90)$ & 0.003 \\
Family history of RA & $1.25(0.60-2.58)$ & 0.55 \\
Duration of RA & $1.00(0.97-1.02)$ & 0.77 \\
UIP on HRCT & $2.44(1.10-5.43)$ & 0.02 \\
FVC \% pred, for 10\% lower than mean 68.7\% & $1.16(1.02-1.34)$ & 0.02 \\
FVC \% pred, 10\% decline any time after baseline & $1.86(1.26-2.75)$ & 0.001 \\
DLco \% pred, for 10\% lower than mean 48.1\% & $1.424(1.18-1.72)$ & 0.0002 \\
DLco \% pred, 10\% decline any time after baseline & $1.77(1.23-2.54)$ & 0.002 \\
CPI & $1.05(1.03-1.08)$ & $<0.0001$ \\
CCP & $1.00(0.99-1.00)$ & 0.61 \\
RF & $1.002(1.000-1.003)$ & 0.02 \\
\hline
\end{tabular}

RA: rheumatoid arthritis; UIP: usual interstitial pneumonia; HRCT: high resolution computed tomography, FVC: forced vital capacity; DLCO: diffusing capacity of the lung for carbon monoxide; CPI: composite physiologic index; CCP: cyclic citrullinated peptide; RF: rheumatoid factor. 
TABLE 3 Multivariate models for predictors of mortality

\section{Variable}

Model 1

Model 2

Model 3

Model 4

Model 5

Model 6

Model 7

Age, year increase over 64.7 $\quad 1.04(1.01-1.07) ; p=0.04 \quad 1.04(1.01-1.08) ; p=0.01 \quad 1.06(1.03-1.10) ; p=0.0002$

Female

$0.77(0.42-1.39) ; p=0.38 \quad 0.92(0.50-1.69), p=0.78$

UIP/HRCT

FVC \% pred, $10 \%$ decline

from baseline

FVC \% pred, $10 \%$ lower

$1.88(0.96-3.69): p=0.07 \quad 2.05(1.03-4.08) ; p=0.04$

$2.03(1.06-3.89) ; p=0.03 \quad 1.88(0.96-3.69)$

$1.62(0.72-3.65) ; p=0.24 \quad 1.62(0.72-3.66) ; p=0.24 \quad 1.56(0.69-3.53) ; p=0.28$

$1.07(1.03-1.11) ; p=0.00021 .07(1.03-1.11) ; p=0.0002 \quad 1.03(0.99-1.06) ; p=0.15$ $0.98(0.52-1.82) ; p=0.94 \quad 0.96(0.52-1.79) ; p=0.91 \quad 0.82(0.44-1.52) ; p=0.52$ $2.15(1.08-4.31) ; p=0.02 \quad 2.19(1.10-4.35) ; p=0.03 \quad 2.03(0.99-4.19) ; p=0.05$ $2.14(0.95-4.86) ; p=0.08 \quad 1.20(0.52-2.74) ; p=0.67 \quad 1.18(0.52-2.70) ; p=0.69$ $2.52(1.78-3.58) ; p<0.00012 .57(1.79-3.70) ; p<0.00012 .61$ (1.82-3.72); $p<0.0001$

$1.36(1.16-1.60) ; p=0.0001 \quad 1.24(1.07-1.44) ; p=0.004 \quad 1.46(1.23-1.73) ; p<0.0001 \quad 1.46(1.23-1.73) ; p<0.0001 \quad 2.33(1.65-3.28) ; p<0.0001$

CPI

432.663

433.894

420.582

411.524

395.869

394.061

$0.99(0.99-1.00) ; p=0.75$

Data are presented as hazard ratio $(95 \% \mathrm{CI})$ and p-value. UIP: usual interstitial pneumonia; HRCT: high resolution computed tomography; FVC: forced vital capacity; CPI: composite physiologic index; AIC: Akaike Information Criterion. ${ }^{\#}$ : age is centred on mean value for cohort; ${ }^{\text {? }}$ : FVC \% pred is centred on mean value for the cohort; ${ }^{+}$: Akaike Information Criterion: lower values signify better model fit. 

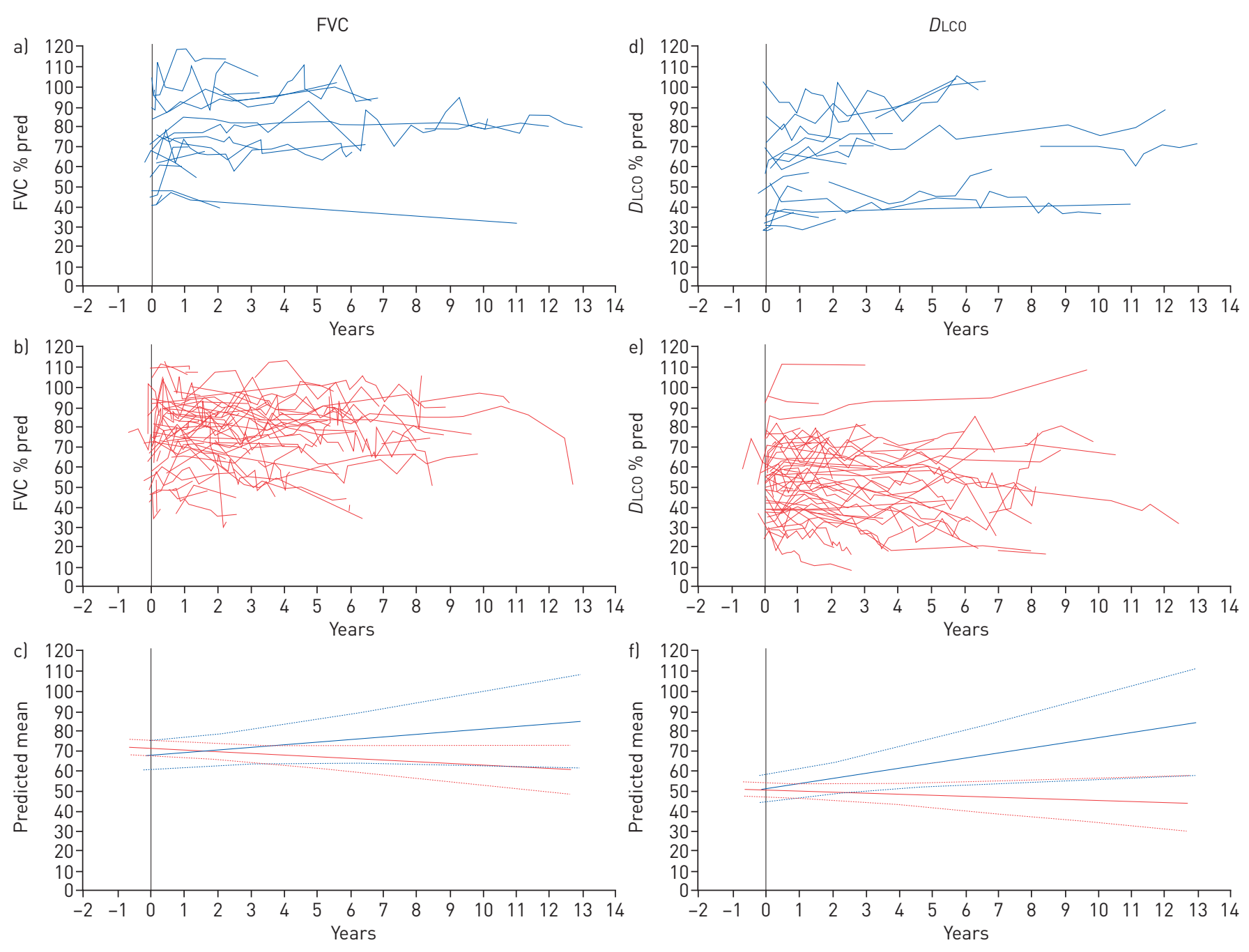

RA-UIP

RA-NSIP

FIGURE 2 a-c) Forced vital capacity (FVC) \% predicted over time by high-resolution computed (HRCT) tomography. pattern. d-f) Diffusing capacity of the lung for carbon monoxide (DLCO) \% pred over time by HRCT pattern. RA: rheumatoid arthritis; UIP: usual interstitial pneumonia; NSIP: nonspecific interstitial pneumonia.

\section{Discussion}

In this study of patients with well-defined RA-ILD, we calculated a median survival of 10.3 years for the cohort as a whole and found a significant difference in survival between subjects with RA-UIP and those with RA-NSIP. Like patients with idiopathic interstitial pneumonia [10], we observed that disease pattern, pulmonary physiology and other variables predict survival. However, in multivariate analyses adjusting for certain key clinical variables (including the confounder, smoking), both baseline and change over time in pulmonary physiology were independent predictors of survival, while baseline HRCT pattern was not. This suggests that RA-ILD patients with greater physiologic impairment (as defined by FVC \% pred, DLCO \% pred or CPI), and those with evidence of disease progression over time (as defined by decline in FVC \% pred or DLCO \% pred) are at increased risk of death, independent of their chest imaging pattern. A similar pattern of results has been seen before. Song et al. [11] found that the progression over time in patients with RA-UIP was variable and depended on age as well as baseline and serial physiology. Bouros et al. [12] observed similar results in patients with another autoimmune disease: systemic sclerosis-related ILD.

ILD is a common manifestation of rheumatoid arthritis, with a prognosis that is generally, but not universally, poor [13]. With $1 \%$ of the US population affected by rheumatoid arthritis, and an estimated $7 \%$ of all deaths in rheumatoid arthritis related to ILD, ILD is not a trivial concern for rheumatoid arthritis patients and the practitioners caring for them [3]. The ability to accurately predict outcome in patients with RA-ILD is important, as such knowledge empowers patients and informs discussions between patients and clinicians around treatment decisions, including referral for transplant evaluation. 
With all available data arising from observational cohort studies [9], confidence in which clinical variables are associated with outcome in RA-ILD could be improved.

In one single-centre study, the median survival after RA-ILD diagnosis was 2.6 years [14]. In another observational study, PARK et al. [7] calculated a median survival of 7.25 years for 18 subjects with RA-UIP, whose survival was worse than for subjects with RA-NSIP; however, the value did not reach statistical significance (HR for RA-UIP=18.95, $\mathrm{p}=0.08$ ). And because of too few events, the median survival for RA-NSIP could not be calculated. Multivariate analyses adjusting for potentially influential predictors of survival were not performed on data from this small subgroup.

Other investigators have observed that age, a rheumatoid arthritis disease activity score [13, 15], UIP-pattern on HRCT, DLCO \% pred at presentation and sex (female protective) $[6,15]$ were significant predictors of mortality. The current study builds on past work from our group: in a prior study, we examined clinical predictors of outcome in RA-ILD defined by lung biopsy and found that age and the presence of any fibrosis on biopsy (UIP, fibrotic NSIP, or unclassifiable fibrosing ILD) predicted mortality [8]. Given the sample size, we did not examine mortality between subjects with UIP and NSIP; however, neither baseline FVC \% pred nor DLCO \% pred were independent predictors of outcome in a model that included the dichotomous variable presence/absence of fibrosis on surgical pathology. In that study, we did not examine the influence of changes in pulmonary physiology over time.

Tobacco exposure plays a major role in the development of anti-CCP positive rheumatoid arthritis and RA-ILD [16-18]. Smokers are more likely to have citrullinated proteins in lung lavage [19], and they have increased rheumatoid arthritis disease activity [20]. As reported by other investigators, in our cohort, smoking was associated with a UIP pattern on HRCT. GILEs et al. [21] demonstrated that, compared with non-smokers, smokers with rheumatoid arthritis had higher numbers of circulating anti-citrullinated protein antibodies and were more likely to have a UIP pattern on HRCT. In most of our multivariate models, smoking conferred a poorer prognosis. This may be related to its ill effects on cardiovascular health in a population already at heightened risk for cardiovascular-related death [22]; however, given emerging data linking cigarette smoking with protein citrullination and the development of rheumatoid arthritis, the effects may not be so straightforward.

In IPF, clinical predictors of mortality are well-known, baseline age [10, 23], pulmonary physiology (FVC $\%$ pred, DLCO \% pred, CPI) [23-25], chest imaging features (extent of reticulation and honeycombing) [26], bronchoalveolar lavage neutrophilia [27], B-type natriuretic peptide level [28], delayed access to a tertiary referral centre [29] and distance covered during a timed walk test (6-min walking distance $(6 \mathrm{MWD})$ ) [30, 31], while change over time in FVC \% pred, DLCO \% pred [32, 33], dyspnoea score [33] and 6MWD $[31,34]$ are all associated with mortality.

Much less is known about prognostic markers for RA-ILD. Although data are emerging, the variable course of IPF has made deriving reliable predictions of IPF behaviour over time for individual patients (as measured by pulmonary physiology) quite challenging. There is a paucity of published data on RA-ILD behaviour over time. Our group recently published results of a retrospective study of patients from a variety of clinical contexts with surgical lung biopsy-proven UIP; thus, no patients with NSIP were included [35]. For the 13 subjects with RA-UIP in that study (three of whom were included in the current study), slopes for both FVC \% pred and DLCO \% pred showed decline over time, similar to the cohort in the current study. Data from the current study show the significant difference in disease behaviour over time for patients with RA-UIP versus those with RA-NSIP.

To make the results of this study clinically relevant, we elected to use HRCT pattern as the main effect variable. Few patients with RA-ILD currently undergo surgical lung biopsy, and HRCT is used primarily to identify the presence of ILD and describe its pattern. AssAYAG et al. [36] found that in RA-ILD, UIP was the dominant histological pattern among patients with rheumatoid arthritis, and UIP on HRCT was 96\% specific but only $45 \%$ sensitive (with a $53 \%$ negative predictive value) for UIP on surgical biopsy; thus, as is the case for patients presenting with an idiopathic interstitial pneumonia, many of the subjects with RA-NSIP in the current study could have had UIP histologic pattern on surgical lung biopsy if one had been done. The key points are these: 1) knowing the HRCT pattern (regardless of the underlying histological pattern) yields important prognostic information; but 2) independent of HRCT pattern (UIP versus NSIP), baseline and change over time in pulmonary physiology variables are strongly associated with prognosis.

There are limitations to this study. All subjects were seen at a tertiary referral centre, thus potentially introducing referral bias and detracting from our ability to generalise these results to the larger rheumatoid arthritis community. All of our subjects had clinically significant ILD with respiratory symptoms, precluding the application of these data to patients with asymptomatic disease (e.g. those with ILD incidentally discovered on chest imaging). With only seven deaths among only 29 subjects in the RA-NSIP group, multivariate survival models (at least the HRCT pattern variable) should be interpreted with 
caution. In certain models, there was a trend toward statistical significance for the HRCT, thus suggesting that if we were able to include a greater number of subjects (and deaths), HRCT pattern would reach statistical significance. We were unable to systematically examine variables (e.g. 6MWD, the presence of pulmonary hypertension, the severity of rheumatoid arthritis joint disease, medication use or serum biomarkers (e.g. rheumatoid factor and anti-CCP)) that may be associated with mortality or disease progression in patients with RA-ILD. Although mixed-effects models are capable of handling certain types of missing data in an unbiased fashion, survivorship bias unavoidably influences longitudinal models: subjects who survive longest contribute more data.

In summary, we identified several clinical predictors of mortality in patients with RA-ILD, including age, HRCT pattern, smoking history and pulmonary physiology. Multivariate survival analysis revealed that baseline and longitudinal changes in physiology were independent predictors of mortality, but in many models in which we controlled for potentially influential variables, baseline HRCT pattern was not. Slopes for both FVC \% pred and DLCO \% pred in patients with RA-UIP were significantly different from patients with RA-NSIP. This information adds to the emerging pool of data on survival and longitudinal disease behaviour in patients with RA-ILD. Our hope is that it can be used to help patients plan for the future, assist clinicians with making decisions around transplant referral and aid investigators as they select clinical endpoints in future interventional treatment trials.

\section{References}

1 Scott DL, Wolfe F, Huizinga TW. Rheumatoid arthritis. Lancet 2010; 376: 1094-1108.

2 Solomon JJ, Brown KK. Rheumatoid arthritis-associated interstitial lung disease. Open Access Rheumatol Res Rev 2012; 4: 21-31.

3 Olson AL, Swigris JJ, Sprunger DB, et al. Rheumatoid arthritis-interstitial lung disease-associated mortality. Am J Respir Crit Care Med 2011; 183: 372-378.

4 Gabbay E, Tarala R, Will R, et al. Interstitial lung disease in recent onset rheumatoid arthritis. Am J Respir Crit Care Med 1997; 156: 528-535.

5 Tanaka N, Kim JS, Newell JD, et al. Rheumatoid arthritis-related lung diseases: CT findings. Radiology 2004; 232: 81-91.

6 Kim EJ, Elicker BM, Maldonado F, et al. Usual interstitial pneumonia in rheumatoid arthritis-associated interstitial lung disease. Eur Respir J 2010; 35: 1322-1328.

7 Park JH, Kim DS, Park IN, et al. Prognosis of fibrotic interstitial pneumonia: idiopathic versus collagen vascular disease-related subtypes. Am J Respir Crit Care Med 2007; 175: 705-711.

8 Solomon JJ, Ryu JH, Tazelaar HD, et al. Fibrosing interstitial pneumonia predicts survival in patients with rheumatoid arthritis-associated interstitial lung disease (RA-ILD). Respir Med 2013; 107: 1247-1252.

9 Assayag D, Lubin M, Lee JS, et al. Predictors of mortality in rheumatoid arthritis-related interstitial lung disease. Respirology 2014; 19: 493-500.

10 Jegal Y, Kim DS, Shim TS, et al. Physiology is a stronger predictor of survival than pathology in fibrotic interstitial pneumonia. Am J Respir Crit Care Med 2005; 171: 639-644.

11 Song JW, Lee HK, Lee CK, et al. Clinical course and outcome of rheumatoid arthritis-related usual interstitial pneumonia. Sarcoidosis Vasc Diffuse Lung Dis 2013; 30: 103-112.

12 Bouros D, Wells AU, Nicholson AG, et al. Histopathologic subsets of fibrosing alveolitis in patients with systemic sclerosis and their relationship to outcome. Am J Respir Crit Care Med 2002; 165: 1581-1586.

13 Koduri G, Norton S, Young A, et al. Interstitial lung disease has a poor prognosis in rheumatoid arthritis: results from an inception cohort. Rheumatology (Oxford) 2010; 49: 1483-1489.

14 Bongartz T, Nannini C, Medina-Velasquez YF, et al. Incidence and mortality of interstitial lung disease in rheumatoid arthritis: a population-based study. Arthritis Rheum 2010; 62: 1583-1591.

15 Dixon WG, Hyrich KL, Watson KD, et al. Influence of anti-TNF therapy on mortality in patients with rheumatoid arthritis-associated interstitial lung disease: results from the British Society for Rheumatology Biologics Register. Ann Rheum Dis 2010; 69: 1086-1091.

16 Baka Z, Buzas E, Nagy G. Rheumatoid arthritis and smoking: putting the pieces together. Arthritis Res Ther 2009; 11: 238.

17 Klareskog L, Stolt P, Lundberg K, et al. A new model for an etiology of rheumatoid arthritis: smoking may trigger HLA-DR (shared epitope)-restricted immune reactions to autoantigens modified by citrullination. Arthritis Rheum 2006; 54: 38-46.

18 Saag KG, Kolluri S, Koehnke RK, et al. Rheumatoid arthritis lung disease. Determinants of radiographic and physiologic abnormalities. Arthritis Rheum 1996; 39: 1711-1719.

19 Makrygiannakis D, Hermansson M, Ulfgren AK, et al. Smoking increases peptidylarginine deiminase 2 enzyme expression in human lungs and increases citrullination in BAL cells. Ann Rheum Dis 2008; 67: 1488-1492.

20 Manfredsdottir VF, Vikingsdottir T, Jonsson T, et al. The effects of tobacco smoking and rheumatoid factor seropositivity on disease activity and joint damage in early rheumatoid arthritis. Rheumatology (Oxford) 2006; 45: $734-740$.

21 Giles JT, Danoff SK, Sokolove J, et al. Association of fine specificity and repertoire expansion of anticitrullinated peptide antibodies with rheumatoid arthritis associated interstitial lung disease. Ann Rheum Dis 2013.

22 Maradit-Kremers H, Nicola PJ, Crowson CS, et al. Cardiovascular death in rheumatoid arthritis: a population-based study. Arthritis Rheum 2005; 52: 722-732.

23 du Bois RM, Weycker D, Albera C, et al. Ascertainment of individual risk of mortality for patients with idiopathic pulmonary fibrosis. Am J Respir Crit Care Med 2011; 184: 459-466.

24 Wells AU, Desai SR, Rubens MB, et al. Idiopathic pulmonary fibrosis: a composite physiologic index derived from disease extent observed by computed tomography. Am J Respir Crit Care Med 2003; 167: 9622-9969. 
Nathan SD, Shlobin OA, Weir N, et al. Long-term course and prognosis of idiopathic pulmonary fibrosis in the new millennium. Chest 2011; 140: 221-229.

26 Lynch DA, Godwin JD, Safrin S, et al. High-resolution computed tomography in idiopathic pulmonary fibrosis: diagnosis and prognosis. Am J Respir Crit Care Med 2005; 172: 488-493.

27 Kinder BW, Brown KK, Schwarz MI, et al. Baseline BAL neutrophilia predicts early mortality in idiopathic pulmonary fibrosis. Chest 2008; 133: 226-232.

28 Song JW, Song JK, Kim DS. Echocardiography and brain natriuretic peptide as prognostic indicators in idiopathic pulmonary fibrosis. Respir Med 2009; 103: 180-186.

29 Lamas DJ, Kawut SM, Bagiella E, et al. Delayed access and survival in idiopathic pulmonary fibrosis: a cohort study. Am J Respir Crit Care Med 2011; 184: 842-847.

30 Lederer DJ, Arcasoy SM, Wilt JS, et al. Six-minute-walk distance predicts waiting list survival in idiopathic pulmonary fibrosis. Am J Respir Crit Care Med 2006; 174: 659-664.

31 Caminati A, Bianchi A, Cassandro R, et al. Walking distance on 6-MWT is a prognostic factor in idiopathic pulmonary fibrosis. Respir Med 2009; 103: 117-123.

32 Flaherty KR, Andrei AC, Murray S, et al. Idiopathic pulmonary fibrosis: prognostic value of changes in physiology and six-minute-walk test. Am J Respir Crit Care Med 2006; 174: 803-809.

33 Collard HR, King TE Jr, Bartelson BB, et al. Changes in clinical and physiologic variables predict survival in idiopathic pulmonary fibrosis. Am J Respir Crit Care Med 2003; 168: 538-542.

34 du Bois RM, Weycker D, Albera C, et al. Six-minute-walk test in idiopathic pulmonary fibrosis: test validation and minimal clinically important difference. Am J Respir Crit Care Med 2011; 183: 1231-1237.

35 Strand MJ, Sprunger D, Cosgrove GP, et al. Pulmonary function and survival in idiopathic vs secondary usual interstitial pneumonia. Chest 2014; 146: 775-785.

36 Assayag D, Elicker BM, Urbania TH, et al. Rheumatoid arthritis-associated interstitial lung disease: radiologic identification of usual interstitial pneumonia pattern. Radiology 2014; 270: 583-588. 